\title{
Application of Empirical Mode Decomposition and Extension Neural Network Type-3 to Feature Diagnosis of Electrocardiograms
}

\author{
Shiue-Der Lu, Meng-Hui Wang, , and Guang-Ci Ye \\ National Chin-Yi University of Technology, Department of Electrical Engineering, \\ No. 35, Lane 215, Sec.1, Chung-Shan Rd., Taiping District, Taichung City 411, Taiwan
}

(Received March 20, 2019; accepted September 9, 2019)

Keywords: extension neural network type-3, chaos theory, empirical mode decomposition, chaos dynamic error scatter map, master and slave chaotic systems

We propose to combine extension neural network type-3 (ENN-3) with the chaos theory and empirical mode decomposition (EMD) for electrocardiography (ECG) identification. ECG signals are measured and captured by the developed hardware measuring circuit and LabVIEW human-machine interface, and the stored ECG data are subjected to EMD at high- and lowfrequency signals. A chaos dynamic error scatter map is formed using master and slave chaotic systems in order to obtain the chaos eye coordinates of a specific ECG signal, and ENN-3 is used for identification. There are 50 research participants in this study; the first half of the data are measured using a signal capturing circuit and a wrist patch-type ECG sensor (patch electrodes), while the second half are provided by Massachusetts Institute of Technology-Beth Israel Deaconess Medical Center (MIT-BIH). Analysis results show that the method proposed in this study has a higher accuracy in the classification of ECG signals, and that the recognition rate is as high as $100 \%$. The recognition result was compared with those of ENN-3, the multilayer neural network, extension method, and ENN. The results showed that ENN-3 has a higher recognition accuracy rate than the other three algorithms, the difference being as much as $8 \%$. Therefore, the autodiagnosis ECG system designed in this study can effectively classify arrhythmia and reduce the high cost of manual identification.

\section{Introduction}

The research on the prevention and diagnosis of heart diseases has been paid increasing attention in recent years. In general, the catheters used and inserted into an artery placed in the wrist play a role in medicine for heart problems. ${ }^{(1)}$ Medical equipment that noninvasively capture the electrocardiography (ECG) signals of the human heart to determine the condition of the heart and to detect and diagnose different heart diseases is mostly used.

In recent years, many researchers have proposed different eigenvalue capture and diagnostic methods, and the ECG disease recognition method is gradually gaining attention. Llamedo and Martinez ${ }^{(2)}$ proposed the use of linear discrimination classification to identify the ECG eigenvalues of the ECG form and RR interval length on the basis of different combinations

*Corresponding author: e-mail: wangmh@ncut.edu.tw

https://doi.org/10.18494/SAM.2019.2381 
of the three types of eigenvalues, where the most efficient feature combination is trained using training data, and the classification system was validated using test data. Kozia et al. ${ }^{(3)}$ identified the PQRST points in the ECG time domain, where the QRS complex wave and QT interval were used as features for identification. It is noteworthy that the eigenvalue extraction methods mostly use the RR interval length and other types of eigenvalue, e.g., the morphological eigenvalue based on wavelet transform, ${ }^{(4)}$ the coefficient based on the Hermite function, ${ }^{(5,6)}$ or features directly extracted from the time and frequency domains of ECG. The classification methods include linear discrimination classification, ${ }^{(7,8)}$ support vector machine $(\mathrm{SVM}),{ }^{(9,10)}$ neural network, ${ }^{(11,12)}$ and fuzzy theory ${ }^{(13,14)}$ methods.

Park et al. ${ }^{(15)}$ proposed the use of statistics and Hermite function methods to extract the eigenvalue of heartbeat signals on the basis of the hierarchical classification method and then SVM to classify ECG signals. Chen et al. ${ }^{(16)}$ used chaotic ECG signals for disease identification and the combination of the correlation dimension and Lyapunov eigenvalues of the chaos theory with neural network learning and identification functions for identification. Zhai and $\operatorname{Tin}^{(17)}$ proposed an ECG-based arrhythmic beat classification system considering beat waveform and beat-to-beat correlation by using the convolutional neural network (CNN). Li and $\mathrm{Li}^{(18)}$ utilized a local deep field method to capture and use the class information hidden in the details of data space for ECG beat classification. Chen et al. ${ }^{(19)}$ presented a novel feature extraction method for the ECG classification based on a combination of projected and dynamic features.

In our study, the input data used are divided into two major parts. One part is composed of arrhythmia data that form the Massachusetts Institute of Technology-Beth Israel Deaconess Medical Center (MIT-BIH) database, which is one of the internationally recognized standard ECG databases and also provides various other resources for research on arrhythmia. ${ }^{(20)}$ The other part is composed of the participants' data of ECG signals measured and extracted using the developed hardware circuit with a wrist patch-type ECG patch electrode sensor. As there are multiple types of feature extraction and calculation is complicated, in order to extract the eigenvalue of the original signal and effectively reduce the measured data volume, we combine extension neural network type-3 (ENN-3) with the chaos theory and empirical mode decomposition (EMD) to enable ECG disease identification. In addition, for future signal extraction, analysis, and identification, we develop a human-machine interface, where realtime measured signals are integrated with the proposed algorithm and displayed on the graphic control screen, using LabVIEW.

In Sect. 2, the overall system architecture will be given. The proposed methods, including EMD, the chaos theory, and ENN-3, will be introduced in Sect. 3. Experimental results will be discussed in Sect. 4. Finally, conclusions will be given in Sect. 5.

\section{System Flow}

\subsection{Overall system architecture}

The application of EMD, ENN-3, and the chaos theory to the ECG recognition system, as proposed in this paper, is divided into three major parts: the measurement and extraction of 
ECG signals; the EMD and feature extraction of chaos eye coordinates of the chaos theory; identification using ENN-3 and the human-machine interface result display. The overall system architecture is shown in Fig. 1.

\section{Step 1: Signal measurement and acquisition}

The first half of the data are from 25 participants. The participants' ECG signals are measured using a hardware circuit, and the data are transmitted to and stored in the server by the DAQ of LabVIEW. The second half of the data are from the MIT-BIH arrhythmia database. The ECG signals are from 50 participants.

\section{Step 2: EMD and chaos theory}

The measured originals ECG signals and MIT-BIH data are used in the intrinsic mode function (IMF) of EMD. The IMF is divided into six layers from high frequencies (IMF1) to low frequencies (IMF6). The chaos dynamic error scatter map is derived from each layer by the master and slave chaotic systems of the chaos theory, and the extracted chaos eye coordinates are used as eigenvalues. Here, we use the root mean square (RMS) value to determine the maximum distance between chaos eyes; thus, IMF6 is selected as the eigenvalue. There are 50 chaos-eye-coordinate eigenvalues.

\section{Step 3: ENN-3 and human-machine interface}

Next, the chaos eye coordinates are classified and identified using ENN-3. Finally, the recognition result is displayed on the developed human-machine interface.

\subsection{ECG signal measurement and extraction hardware circuit}

Figure 2 shows the physical circuit for ECG signal measurement and extraction, including the electrode patch, an extraction circuit composed of a preamplifier, a power amplifier, highand low-pass filters, and a DAQ card for analog-to-digital signal conversion. Finally, the signal is exported to the human-machine interface designed using LabVIEW.

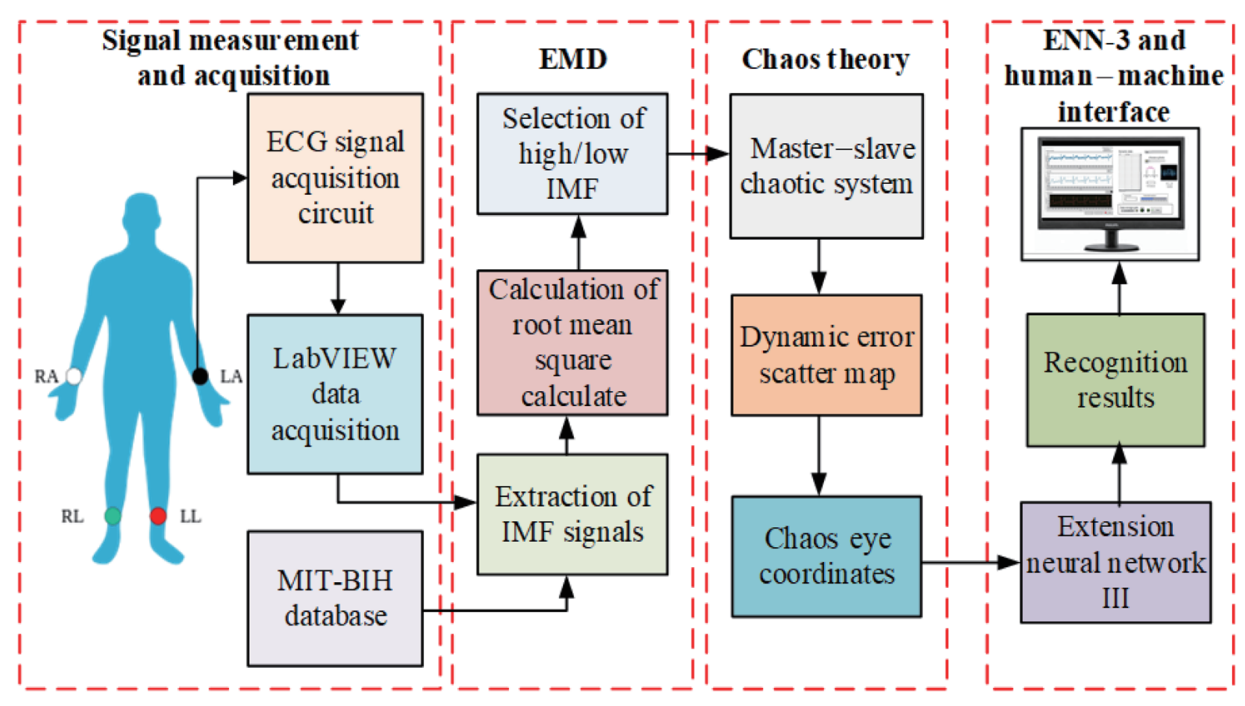

Fig. 1. (Color online) System architecture flowchart. 


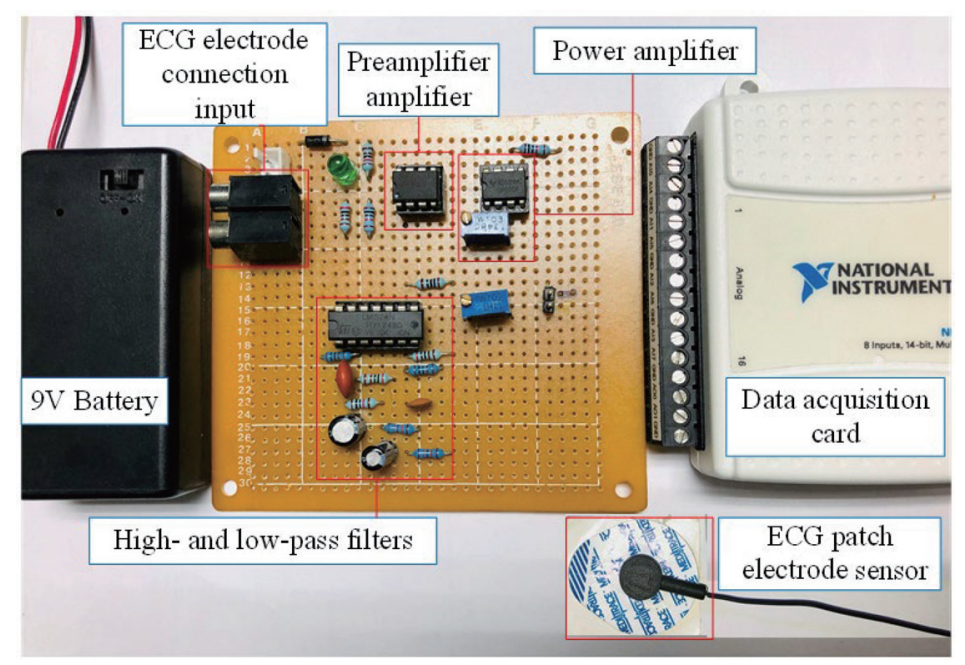

Fig. 2. (Color online) ECG signal measurement and extraction hardware circuit diagram.

Preamplifier: As some measured signals are very weak, the ECG signal is usually amplified by a preamplifier circuit. In this study, we use the AD620 instrument amplifier, which is a high-gain DC coupling amplifier characterized by a differential input, a single-ended output, a high input impedance, and a high common-mode rejection ratio (CMRR). The accuracy of the amplified signal is high, the noise is low, the operation voltage is low, and it is easy to use.

Filter: The ECG signal is amplified by AD620, and then, the measurement range is designed to be in a certain frequency range using a filter. In this study, a second-order Butterworth filter, ${ }^{(21)}$ where the cutoff frequencies of low-pass and high-pass filters are designed as 100 and $0.05 \mathrm{~Hz}$, respectively, is used.

Power amplifier: After the signal is processed by the preamplifier and filter, the signal must be amplified by the power amplifier circuit. We use the operational amplifier LM324 IC for Stage 2 circuit voltage gain.

\section{Proposed Methods}

\subsection{EMD}

The $\mathrm{EMD}^{(22,23)}$ for signal preprocessing, as proposed in this paper, can effectively separate the frequency components of a signal from the time curve in the form of IMF, and the components of the original signal in different frequency bands are traced out by reconstruction. The important information contained in the original signal can be extracted during the course of decomposition. The basic equation is described below. If the time series signal is $x(t)$, all the extreme points on $x(t)$ are determined and joined by the curve, where the upper and lower envelope curves of signal $x(t)$ are set as $u_{0}(t)$ and $v_{0}(t)$, respectively. The average curve of the upper and lower envelopes is expressed as 


$$
m_{0}(t)=\frac{1}{2}\left[u_{0}(t)+v_{0}(t)\right]
$$

After $m_{0}(t)$ is subtracted from $x(t), h_{1}(t)=x(t)-m_{0}(t)$, the function may or may not be an IMF component. In general, it does not satisfy the condition for IMF.

$$
\left\{\begin{aligned}
m_{1}(t) & =\frac{1}{2}\left[u_{1}(t)+v_{1}(t)\right] \\
h_{2}(t) & =h_{1}(t)-m_{1}(t) \\
& \vdots \\
m_{k-1}(t) & =\frac{1}{2}\left[u_{k-1}(t)+v_{k-1}(t)\right] \\
h_{k}(t) & =h_{k-1}(t)-m_{k-1}(t)
\end{aligned}\right.
$$

In the aforesaid decomposition process, when a certain standard is reached after multiple repetitions, $h_{k}(t)$ becomes the first IMF of the original signal, which is set as $y_{1}(t)$, and the rest of the signal is set as $r_{1}(t)$. Then,

$$
\left\{\begin{array}{l}
y_{1}(t)=h_{k}(t) \\
r_{1}(t)=x(t)-y_{1}(t)
\end{array}\right.
$$

where $y_{1}(t)$ is the first component obtained by processing the original data, as well as the highest-frequency component in the $x(t)$ of the original signal; $r_{1}(t)$ is the corresponding residual component. $\quad r_{1}(t)$ in the remainder is used as the new original signal in repeating the aforesaid process, and the EMD continues. The second IMF $y_{2}(t)$ can be calculated, the process is repeated $n$ times until the remainder becomes a monotonic signal or its value becomes smaller than the present value, upon which decomposition is completed, and the number $n \operatorname{IMF} y_{n}(t)$ and residual component $r_{n}(t)$ are obtained.

$$
\left\{\begin{array}{c}
r_{1}(t)-y_{2}(t)=r_{2}(t) \\
\vdots \\
r_{n-1}(t)-y_{n}(t)=r_{n}(t)
\end{array}\right.
$$

The important information in the fault signal can be extracted by repeated EMD and the noise interference can be eliminated.

\subsection{Chaos theory}

The chaos theory proposed by the American meteorologist Edward Norton Lorenz discusses the unsteady behavior of a nonlinear dynamic system. The chaotic attractor results in an orderly, but nonperiodic kinematic trajectory, of the signal generated by the chaos theory, 
and this trajectory can change the result markedly through subtle changes. ${ }^{(24)}$ Therefore, it is applicable to the large data volume of the original signal and to values that change in a small range.

The chaotic system is divided into a master system and a slave system, expressed as Eqs. (5) and (6), respectively. The chaos dynamic error resulting from the difference between the values of the two systems leads to different operation trajectories of the master and slave systems. In the engineering domain, when the master system is tracked by the slave system and the two system operation trajectories are coordinated gradually by the controller, the system is known as a chaotic synchronization system. ${ }^{(25)}$

$$
\begin{gathered}
S_{\text {master }}=\left\{\begin{array}{c}
\dot{x}_{1}=f_{1}\left(x_{1}, x_{2}, x_{3}, \cdots, x_{n}\right) \\
\dot{x}_{2}=f_{2}\left(x_{1}, x_{2}, x_{3}, \cdots, x_{n}\right) \\
\vdots \\
\dot{x}_{n}=f_{n}\left(x_{1}, x_{2}, x_{3}, \cdots, x_{n}\right)
\end{array}\right. \\
S_{\text {slave }}=\left\{\begin{array}{c}
\dot{y}_{1}=f_{1}\left(y_{1}, y_{2}, y_{3}, \cdots, y_{n}\right) \\
\dot{y}_{2}=f_{2}\left(y_{1}, y_{2}, y_{3}, \cdots, y_{n}\right) \\
\vdots \\
\dot{y}_{n}=f_{n}\left(y_{1}, y_{2}, y_{3}, \cdots, y_{n}\right)
\end{array}\right.
\end{gathered}
$$

Here, $f_{1}$ is a nonlinear function and Eq. (6) is subtracted from Eq. (5) to obtain the following dynamic error equation.

$$
\left\{\begin{array}{c}
\dot{e}_{1}=f_{1}\left(y_{1}, y_{2}, y_{3}, \cdots, y_{n}\right)-f_{1}\left(x_{1}, x_{2}, x_{3}, \cdots, x_{n}\right) \\
\dot{e}_{2}=f_{1}\left(y_{1}, y_{2}, y_{3}, \cdots, y_{n}\right)-f_{1}\left(x_{1}, x_{2}, x_{3}, \cdots, x_{n}\right) \\
\vdots \\
\dot{e}_{n}=f_{n}\left(y_{1}, y_{2}, y_{3}, \cdots, y_{n}\right)-f_{n}\left(x_{1}, x_{2}, x_{3}, \cdots, x_{n}\right)
\end{array}\right.
$$

The Lorenz master-slave chaos system used in this study is expressed as

$$
\begin{gathered}
L_{\text {master }}=\left\{\begin{array}{l}
\dot{x}_{1}=\alpha\left(x_{2}-x_{1}\right) \\
\dot{x}_{2}=\beta x_{1}-x_{1} x_{3}-x_{2}, \\
\dot{x}_{3}=x_{1} x_{2}-\gamma x_{3}
\end{array}\right. \\
L_{\text {slave }}=\left\{\begin{array}{l}
\dot{y}_{1}=\alpha\left(y_{2}-y_{1}\right) \\
\dot{y}_{2}=\beta y_{1}-y_{1} y_{3}-y_{2} . \\
\dot{y}_{3}=y_{1} y_{2}-\gamma y_{3}
\end{array}\right.
\end{gathered}
$$

Equation (9) is subtracted from Eq. (8) to obtain the dynamic error equation of the Lorenz master-slave chaos system expressed as 


$$
\left[\begin{array}{l}
\dot{e}_{1} \\
\dot{e}_{2} \\
\dot{e}_{3}
\end{array}\right]=\left[\begin{array}{ccc}
-\alpha & \alpha & 0 \\
\beta & -1 & 0 \\
0 & 0 & -\gamma
\end{array}\right]\left[\begin{array}{l}
e_{1} \\
e_{2} \\
e_{3}
\end{array}\right]+\left[\begin{array}{c}
y_{2} y_{3}-x_{2} x_{3} \\
-y_{1} y_{3}+x_{1} x_{3} \\
y_{1} y_{2}-x_{1} x_{2}
\end{array}\right],
$$

where $x$ is the master system whose initial value is set as $0 . y$ is the slave system whose initial value is the ECG signal value. The Lorenz primer coefficients are $\alpha=10, \beta=28$, and $\gamma=(-8 / 3)$.

\subsection{ENN-3}

ENN-3 ${ }^{(26)}$ is an expansion of ENN-2, ${ }^{(27)}$ which is a combination of a neural network ${ }^{(28)}$ and the extension theory ${ }^{(29)}$ introduced in our previous paper. The extension theory provides a distance parameter (DP) for classification, while the neural network uses the features of parallel computation and learning capability.

\subsubsection{ENN-3 architecture}

ENN-3 has been successfully employed in ECG diagnosis. A schematic of the structure of ENN-3 is displayed in Fig. 3. This network consists of two connection values between the input and competitive layers. One of the connections denotes the lower bound and the other connection shows the upper bound. The output nodes $w_{m j}^{L}$ and $w_{m j}^{U}$ provide the connections between the $j$ th and $m$ th input nodes. The main goal of the competitive layer is to partition the subclusters of input features by unsupervised learning. The output layer utilizes the logical 'OR' to combine similar subclusters on the basis of trained data when the clustering procedure in the competitive layer is convergent.

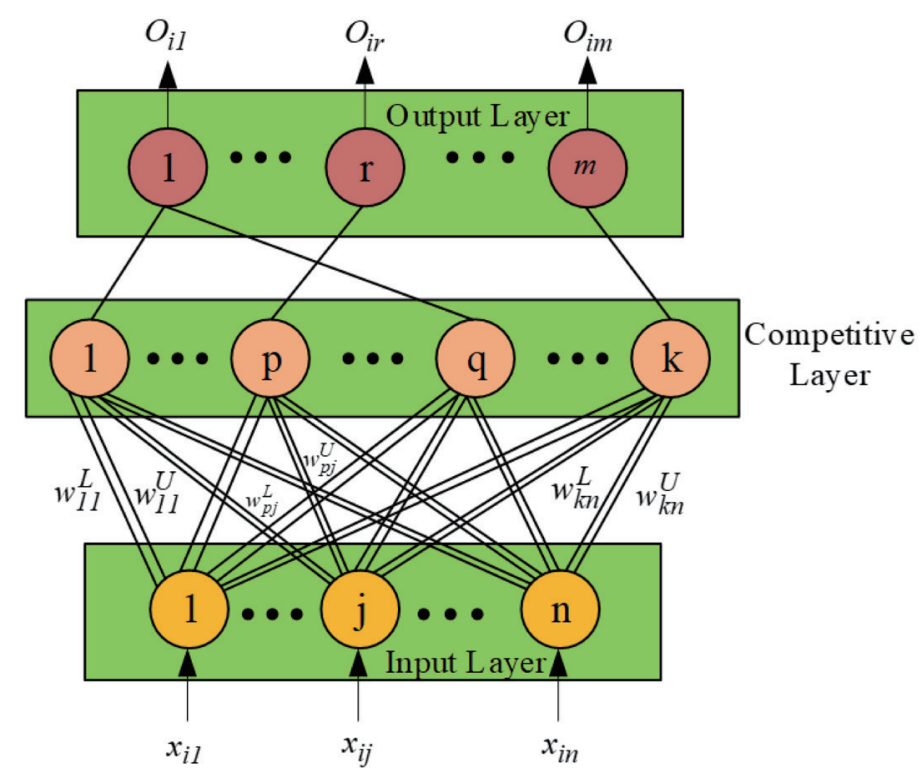

Fig. 3. (Color online) Structure of ENN-3. 


\subsubsection{ENN-3 algorithm}

The ENN-3 algorithm examines both the supervised and unsupervised learning methods. In the first phase, ENN-3 employs the unsupervised learning method to generate subclusters in the competitive layer. A threshold called the DP $\lambda$ and an extension distance (ED) function are used to dominate the clustering process. $\lambda$ is applied to measure the distance between the cluster center and the desired boundary. A pattern is selected as the center of the first cluster, and the initial weights of the first cluster can be calculated from the center and the desired DP $\lambda$. Then, the next pattern is compared and clustered with the first cluster if its distance is less than the threshold. Otherwise, it constitutes the center of a new cluster. This process is repeated for all patterns until a stable or desired cluster formation occurs. In the second phase, ENN-3 utilizes the supervised learning method to combine similar clusters on the basis of the target output between the competitive and output layers. The weights between the competitive and output layers can be set to 1 . A number of variables must be defined before examining the learning process, as shown in Table 1.

The detailed ENN-3 algorithm is outlined below.

Step 1: After setting it, the desired DP is used to measure the distance between the center of the cluster and the desired boundary.

Step 2: The first pattern is produced and $M_{1}=1$. The center coordinates and weights of the first cluster are then calculated.

$$
\begin{gathered}
k=1 \\
Z_{k}=X_{k} \Rightarrow\left\{z_{k 1}, z_{k 2}, \ldots, z_{k n}\right\}=\left\{x_{k 1}, x_{k 2}, \ldots, x_{k n}\right\} \\
w_{k j}^{L}=z_{k j}-\lambda \text { for } j=1,2, \ldots, n \\
w_{k j}^{U}=z_{k j}+\lambda \text { for } j=1,2, \ldots, n
\end{gathered}
$$

Step 3: The input pattern vectors are read by setting $i=2$. Go to the next step.

Step 4: Read the $i$ th input pattern $X_{i} \Rightarrow\left\{x_{i 1}, x_{i 2}, \ldots, x_{i n}\right\}$ before calculating the extension distance $E D_{m}$ between $X_{i}$ and the current $m_{\text {th }}$ cluster center as follows.

Table 1

Parameters of ENN-3 algorithm.

\begin{tabular}{lc}
\hline Variable & Description \\
\hline$X_{i}$ & $i$ th pattern \\
$x_{i j}$ & $j$ th features of $i$ th input pattern \\
$Z_{k}$ & center of cluster $k$ \\
$\lambda$ & distance parameter \\
$N_{p}$ & total number of input patterns \\
$n$ & number of features \\
$k$ & number of existing clusters \\
$M_{k}$ & number of patterns belonging to cluster $k$ \\
$O_{k}^{c}$ & output of $k$ th node in competitive layer \\
\hline
\end{tabular}




$$
E D_{m}=\sum_{j=1}^{n}\left[\frac{\left|x_{i j}-z_{m j}\right|-\frac{\left(w_{m j}^{U}-w_{m j}^{L}\right)}{2}}{\left|\frac{\left(w_{m j}^{U}-w_{m j}^{L}\right)}{2}\right|}+1 \text { for } m=1,2, \ldots, k\right.
$$

Step 5: Find $E D_{p}$ as follows.

$$
E D_{p}=\min \left\{E D_{m}\right\} \text { for } m=1,2, \ldots, k
$$

Step 6: If $E D_{p}>n$, then create a new cluster center. According to the definition of the proposed extension distance, if $x$ lies in the interval, then the distance is smaller than 1 . Thus, if $X_{i}=\left\{x_{i 1}, x_{i 2}, \ldots, x_{i n}\right\}$ has $n$ features in the clustering process, $E D_{p}>n$ indicates that $X_{i}$ does not belong to the $p_{\text {th }}$ cluster. Then, a new cluster center will be created.

$$
\begin{gathered}
k=k+1 \\
Z_{k}=X_{k} \Rightarrow\left\{z_{k 1}, z_{k 2}, \ldots, z_{k n}\right\}=\left\{x_{i 1}, x_{i 2}, \ldots, x_{i n}\right\} \\
w_{k j}^{L}=z_{k j}-\lambda \text { for } j=1,2, \ldots, n \\
w_{k j}^{U}=z_{k j}+\lambda \text { for } j=1,2, \ldots, n \\
O_{k}^{c}=1 ; O_{m}^{c}=0 \text { for all } m \neq k \\
M_{k}=1
\end{gathered}
$$

Otherwise, the pattern $X_{i}$ belongs to cluster $s$, and the weights are updated as follows.

$$
\begin{gathered}
w_{s j}^{U(\text { new })}=w_{s j}^{U(\text { old })}+\frac{1}{M_{s}+1}\left(x_{i j}+z_{s j}^{\text {old }}\right) \\
w_{s j}^{L(\text { new })}=w_{s j}^{L(\text { old })}+\frac{1}{M_{s}+1}\left(x_{i j}+z_{s j}^{\text {old }}\right) \\
z_{s j}^{\text {new }}=\frac{w_{s j}^{U(\text { new })}+w_{s j}^{L(\text { new })}}{2} \text { for } j=1,2, \ldots, n \\
O_{s}^{c}=1 ; O_{p}^{c}=0 \text { for all } p \neq s \\
M_{p}=M_{p}+1
\end{gathered}
$$

Note that only one node should be active to demonstrate a classification of the input pattern and the output of other nodes should be set to nonactive. 
Step 7: Change the input pattern $X_{i}$ from cluster " $o$ " (the old one) to cluster " $k$ " (the new one). Then, the weights and center of cluster " $O$ " are updated as follows.

$$
\begin{gathered}
w_{o j}^{U(\text { new })}=w_{o j}^{U(\text { old })}+\frac{1}{M_{o}}\left(x_{i j}+z_{o j}^{o l d}\right) \\
w_{o j}^{L(\text { new })}=w_{o j}^{L(\text { old })}+\frac{1}{M_{o}}\left(x_{i j}+z_{o j}^{o l d}\right) \\
z_{o j}^{\text {new }}=\frac{w_{o j}^{U(\text { new })}+w_{o j}^{L(\text { new })}}{2} \text { for } j=1,2, \ldots, n \\
M_{o}=M_{o}+1
\end{gathered}
$$

Step 8: Set $i=i+1$ and repeat Steps 4 to 8 iteratively until all the patterns have been compared with the existing subclusters. If the clustering process has converged, go to the next step; otherwise, return to Step 4.

Step 9: Use the logical "Or" to obtain similar subclusters with similar output in accordance with the trained data. The functions of this layer are shown below.

$$
\begin{gathered}
N E T_{r}=\sum W_{r p} O_{p}^{c} \\
O_{r}=\min \left(1, N E T_{r}\right) \text { for } r=1,2, \ldots, m
\end{gathered}
$$

\section{Experimental Results}

\subsection{Chaotic dynamic error scatter diagram}

To validate the performance and accuracy of disease identification using the methods proposed in this paper, heartbeat rate data from 50 participants aged between 20-50-yearold males and females, were tested. Half of the test samples selected from the MIT-BIH ${ }^{(30)}$ arrhythmia database were used to obtain disease identification data. The participants' heartbeat rates were further categorized into four classes, 25 being classified as normal beat (NB), 6 as left bundle branch beat (LBBB), 19 as right bundle branch beat (RBBB), and 2 as atrial premature contraction beat (APCB).

The measured and collected ECG data were used for the IMF of EMD, as shown in Fig. 4, where the value of IMF6 after IMF was calculated using the Lorenz master-slave chaos system of the chaos theory to obtain the chaos dynamic error scatter map. Figure 5 shows the chaos dynamic error scatter map of the heartbeat class LBBB, where each scatter diagram has two chaos eyes. There are four coordinate values, $\mathrm{C} 1$ to $\mathrm{C} 4$. In this study, we used the $y$-coordinate values of the left and right chaos eyes $(\mathrm{C} 1$ and $\mathrm{C} 3)$ as new eigenvalues and obtained 50 chaos eye coordinate eigenvalues. The distribution of the chaos eye coordinates of the four heartbeat classes is shown in Fig. 6. 


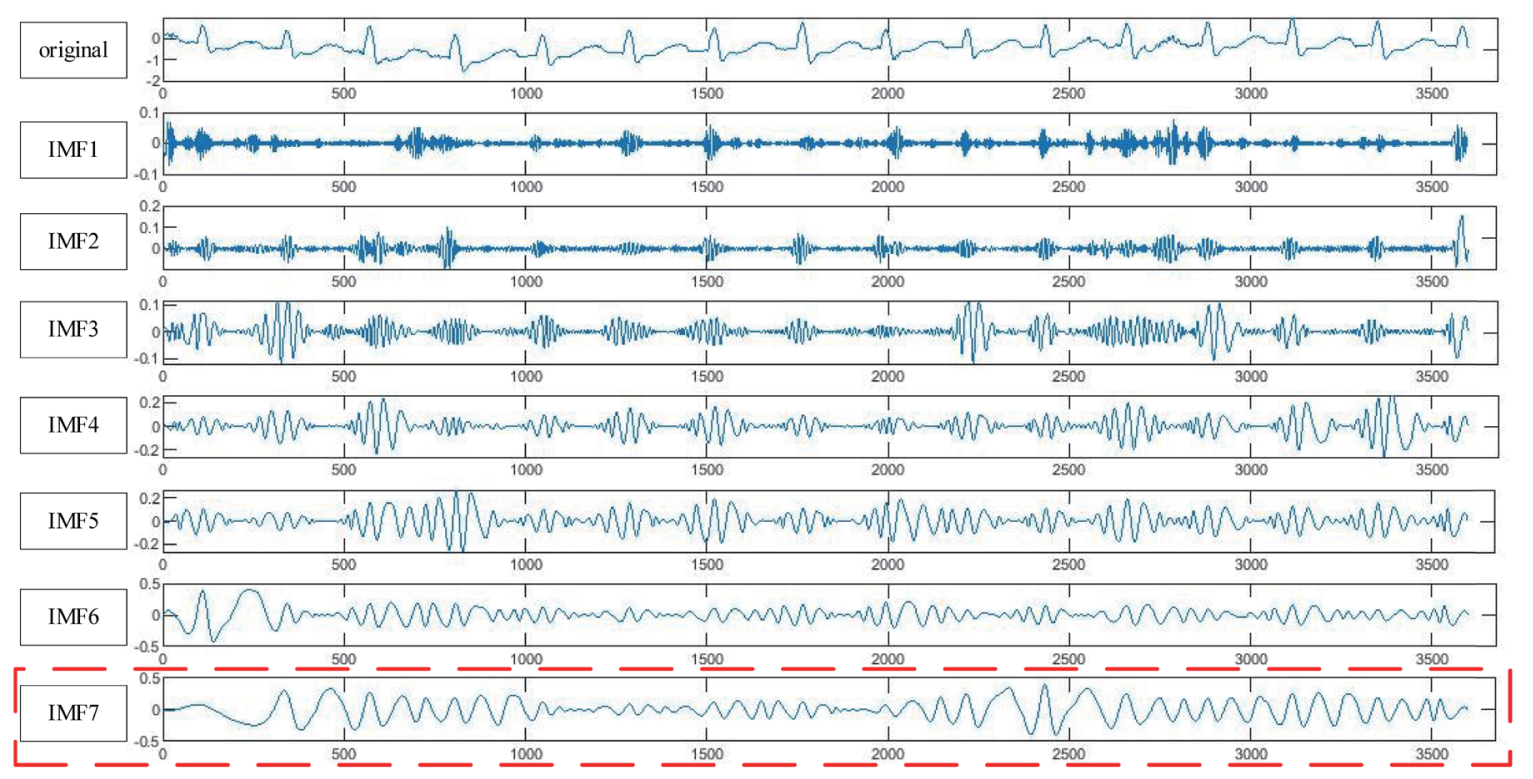

Fig. 4. (Color online) IMF of EMD.

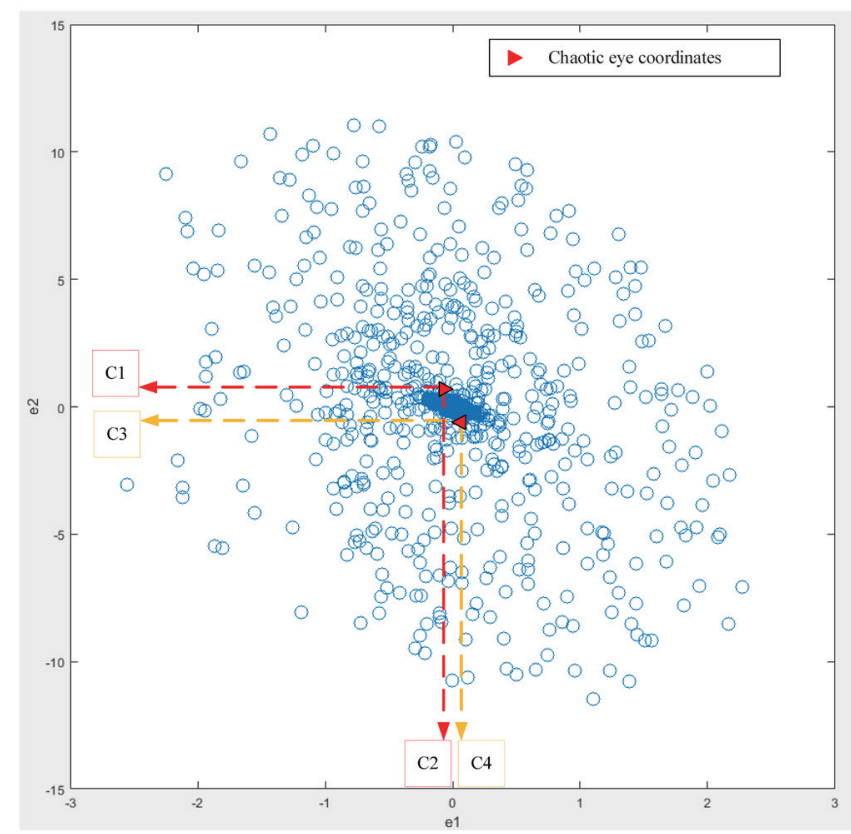

Fig. 5. (Color online) LBBB chaos dynamic error scatter map.

\subsection{ENN-3 identification classification results}

The obtained 50 chaos eye coordinate eigenvalues and ENN-2 recognition results with $\lambda=$ 0.25 are shown in Fig. 7. ENN-3 was further employed for clustering, as shown in Fig. 8. The recognition accuracy is as high as $100 \%$. 


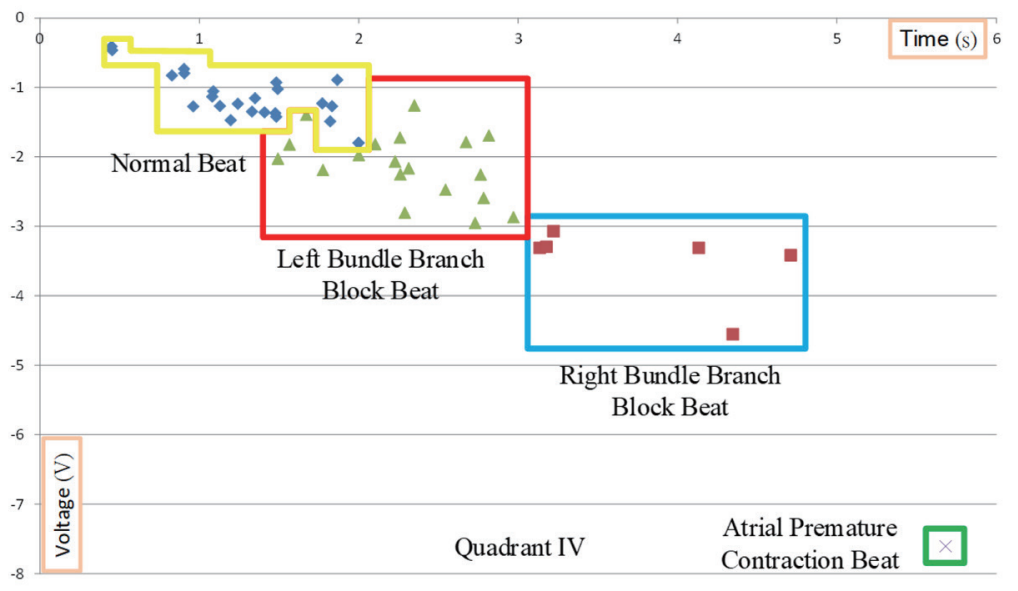

Fig. 6. (Color online) Distribution diagram of $Y$-coordinate values of left and right chaos eyes.

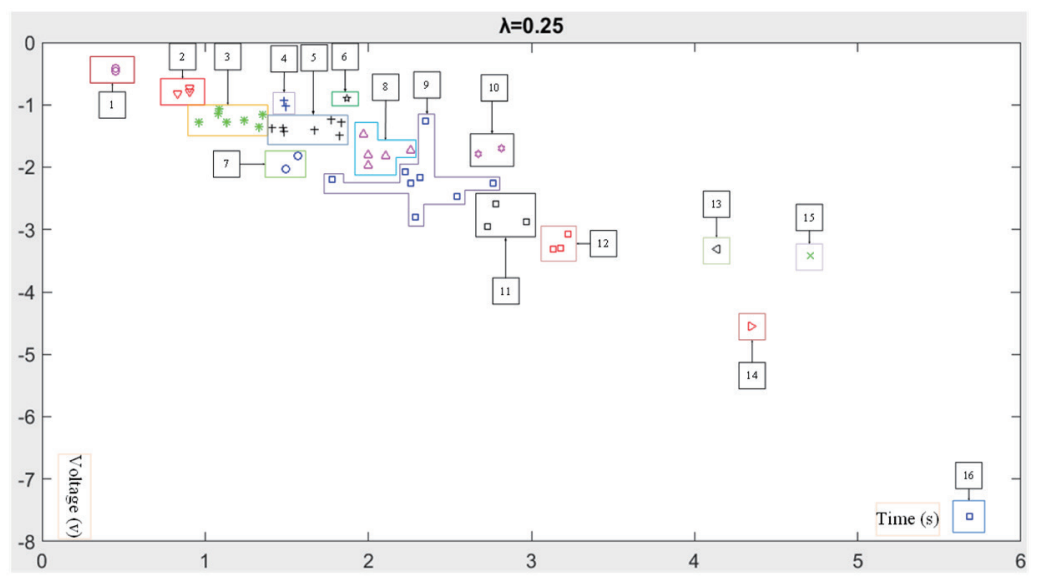

Fig. 7. (Color online) Distribution diagram of ENN-2 recognition results $(\lambda=0.25)$.

The neural networks of other architectures were tested using two eigenvalue inputs and seven fault-type outputs. Only the hidden layer architecture setting was adjusted for 6,8 , and 10 hidden layers and tested. In terms of learning and overall recognition rates, the recognition accuracy rate for 10 hidden layers was the highest at $83.29 \%$. The recognition result was compared with those of ENN-3, the extension method, and ENN, as shown in Table 2. The recognition rate of ENN-3 was the highest (100\%), that of ENN was the second highest (92\%), and that of the extension theory was $92 \%$. The results showed that ENN-3 has a higher recognition accuracy rate than the other three algorithms, the difference being as high as $8 \%$.

\subsection{Human-machine interface signal measurement and result display}

Figure 9 shows the display menu of the human-machine interface developed using LabVIEW graphic control software and the algorithm written by MATLAB, the ECG measurement signal, the dynamic error scatter diagram obtained using the chaos theory after EMD, the numerical display of chaos eye coordinates, and the classification recognition result of ENN-3. 


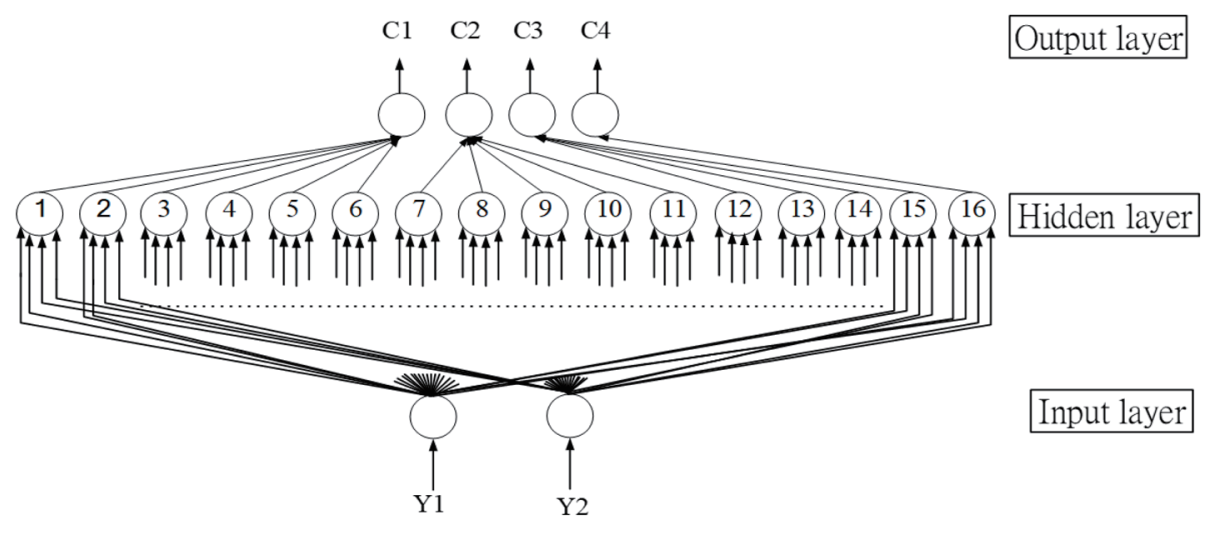

Fig. 8. ENN-3 classification result.

Table 2

Recognition results of ENN-3 and other algorithms.

\begin{tabular}{lccc}
\hline Algorithm & Training time & Learning rate (\%) & Accuracy rate (\%) \\
\hline ENN-3 & 5 & & 100 \\
ENN & 10 & 88 & 92 \\
Multilayer neural network (2-6-7) & 10000 & 99 & 83.25 \\
Multilayer neural network (2-8-7) & 10000 & 99 & 83.27 \\
Multilayer neural network (2-10-4) & 10000 & 99 & 83.29 \\
Extension method & & & 92
\end{tabular}

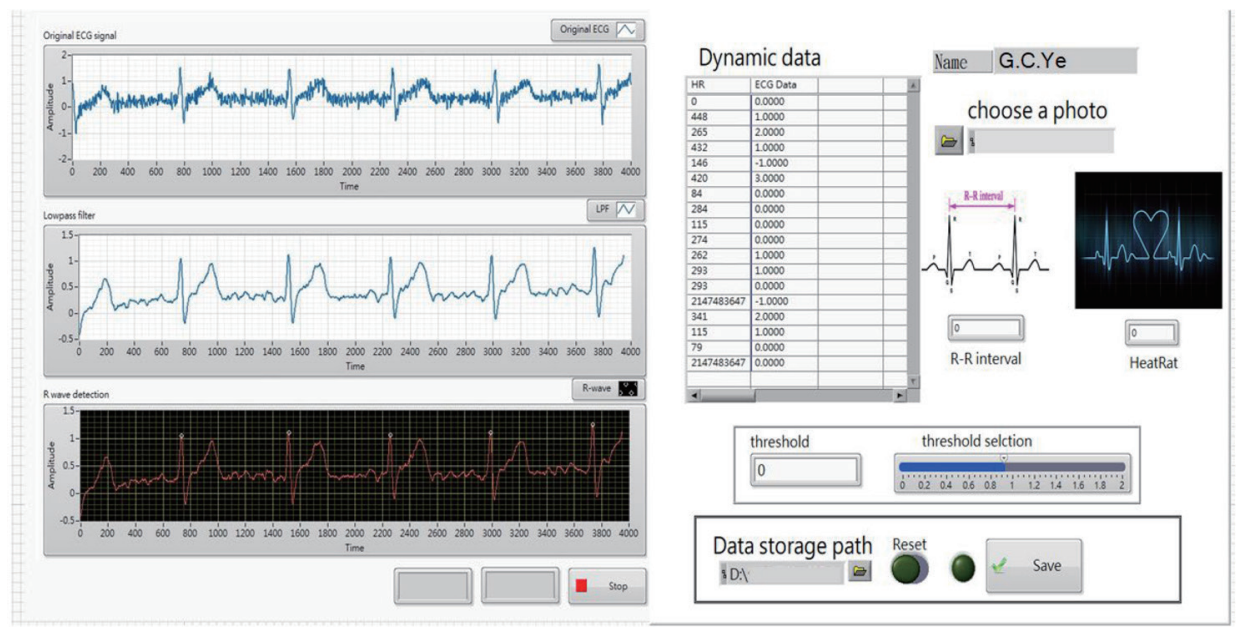

Fig. 9. (Color online) Human-machine interface display of ECG.

\section{Conclusions}

In this study, we developed the software and hardware of an ECG recognition system. The designed ECG measurement and extraction circuit architecture with an ECG patch electrode sensor is simple and includes the developed LabVIEW human-machine interface; the participants' heart status can be known from analytical results. In addition, signals are measured and extracted using the ECG hardware circuit, the stored data are processed by EMD 
to form a dynamic error scatter diagram using the master and slave chaotic systems of the chaos theory, and the chaos eye is used as the eigenvalue and classified by ENN-3 in order to diagnose the subject's heart status. The recognition accuracy is as high as $100 \%$. According to experimental results, the master and slave chaotic systems are used in feature extraction to generate a chaos dynamic error scatter map and chaos eyes. The left and right chaos eye coordinates are used as eigenvalues, and the feature extraction in the time domain of the traditional ECG is greatly reduced in order to reduce the computing time and system complexity of the identification system and achieve efficient diagnosis.

\section{References}

1 S. S. Ahrabi, M. Shojafar, H. K. Esfeh, and A. Abraham: Int. Conf. Hybrid Intelligent Systems (2014) 315.

2 M. Llamedo and J. P. Martinez: IEEE Biomed. Eng. 58 (2011) 3.

3 C. Kozia, R. Herzallah, and D. Lowe: 2018 12th Int. Conf. Signal Processing and Communication Systems (ICSPCS) (IEEE, 2018) 1. https://doi.org/10.1109/ICSPCS.2018.8631760

4 A. Rabee and I. Barhumi: Int. Conf. Information Science, Signal Processing and their Applications (2012) 1319. https://ieeexplore.ieee.org/document/6310497

5 W. K. Lei, B. N. Li, M. C. Dong, and B. B. Fu: Artif. Intell. 31 (2007). https://ieeexplore.ieee.org/ document $/ 4659298$

6 P. de Chazal, M. O’Dwyer, and R. Reilly: IEEE Biomed. Engi. 51 (2004) 7.

7 J. S. Wang, W. C. Chiang, Y. L. Hsu, and Y. T. C. Yang: Elsevier Neurocomputing. 116 (2013) 38. https://www. sciencedirect.com/science/article/pii/S0925231212006960

8 Y. C. Yeh, W. J. Wang, and C. W. Chiou: Elsevier Measurement 42 (2009) 778. https://www.sciencedirect.com/ science/article/pii/S0263224109000050

9 N. Cristianini and J. S.Taylor: An Introduction to Support Vector Machines and Other Kernel-based Learning Methods (Cambridge University Press, Cambridgeshire, 2000).

10 K. Park, B. Cho, D. Lee, S. Song, J. Lee, Y. Chee, I. Kim, and S. Kim: Comput. Cardiol. 35 (2008) 229. https:// ieeexplore.ieee.org/document/4749019?arnumber=4749019\&tag=1

11 N. Jin and D. Liu: IEEE Transa. 19 (2008) 3.

12 K. C. Chen and P. C. Chien: IEEE Global Conf. (2017) 1. https://ieeexplore.ieee.org/document/8229377

13 R. Silipo, W. Zong, and M. Berthold: Comput. Cardiol. 26 (1999) 679. https://ieeexplore.ieee.org/ document $/ 826062$

14 G. R. Naik and K. A. Reddy: IEEE Biomed. Eng. (2016) 138. https://ieeexplore.ieee.org/abstract/ document $/ 8057455$

15 K. Park, B. Cho, D. Lee, S. Song, J. Lee, Y. Chee, I. Kim, and S. Kim: Comput. Cardiol. 35 (2008) 229. https:// ieeexplore.ieee.org/document/4749019

16 C. K. Chen, C. L. Lin, and Y. M. Chiu: Industrial Electronics and Applications (2011).

17 X. Zhai and C. Tin: IEEE Access 6 (2018) 27465.

18 W. Li and J. Li: IEEE Sens. J. 18 (2017) 1656.

19 S. Chen, W. Hua, Z. Li, J. Li, and X. Gao: Elsevier Biomedical Signal Processing and Control. 31 (2017) 165. https://www.sciencedirect.com/science/article/pii/S1746809416300908

20 MIT-BIH Arrhythmia Database: http://www.physionet.org/physiobank/database/

21 G. Bianchi and R. Sorrentino: Electronic Filter Simulation \& Design (McGraw-Hill, New York, 2007) p. 17.

22 D. Yang, M.Z. Qin, and B. Xu: Int. J. Signal Proc. 8 (2015) 2.

23 M. A. Özdemir and A. Akanc: IEEE Medical Technologies National Congress (2018).

24 E. N. Lorenz: J. Atmos. Sci. 20 (1963).

25 H. T. Yau and M. H. Wang: IET Renewable Power Gener. 9 (2015) 6.

26 M. H. Wang: Lect. Notes Comput. Sci. 3496 (2005) 503.

27 M. H. Wang: IEEE Trans. Neural Networks 16 (2005) 1352.

28 M. H. Wang and C. P. Hung: Neural Network 16 (2003) 5.

29 R. O. Duda and P. E. Hart: Pattern Classification and Scene Analysis (Wiley, Hoboken, 1973).

30 A. Rabee and I. Barhumi: Signal Processing and Their Applications (2012). 